\title{
AD-0045 - Assessment of 10 years risk of type 2 diabetes among people of Uzbek nationality: screening results
}

\author{
Ismailov S. ${ }^{1}$, Alieva A. ${ }^{2}$; Rakhimova G. ${ }^{3}$ \\ ${ }^{1}$ Tashkent Pediatric Medical Institute \\ ${ }^{2}$ Republican Specialized Scientific-Practical Medical Centre of Endocrinology \\ ${ }^{3}$ Tashkent Institute for Doctors' Postgraduate Education
}

\section{Diabetes Screening Strategy}

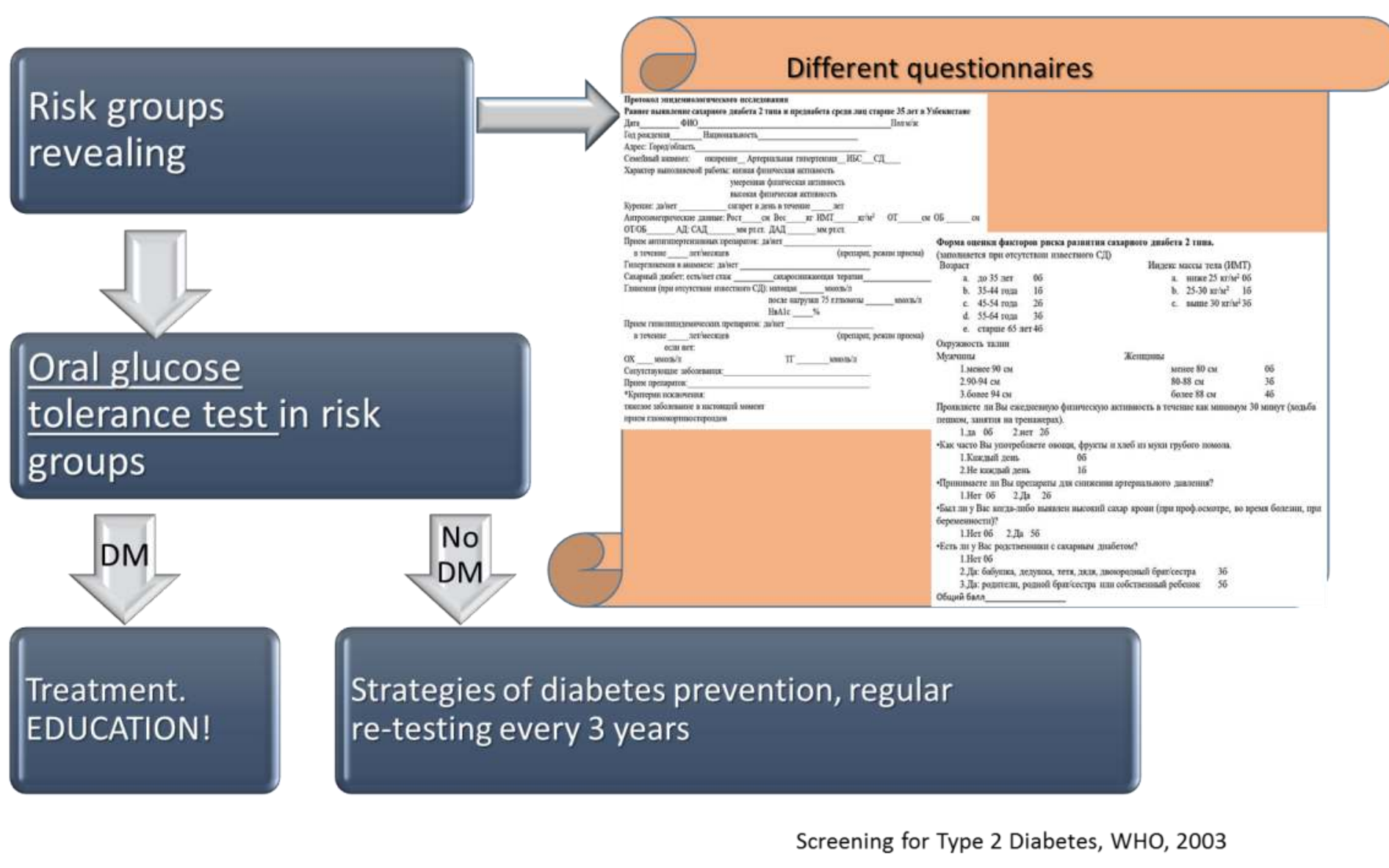

Background and aim: there are several scales for assessment of risk of type $2 \mathrm{DM}$ at the first stage of its active screening, one of the most popular are based on the FINDRISK questionnaire which is easy and has no need for complicated and expensive measurements. The aim of our study was to assess 10 years risk of type $2 \mathrm{DM}$ among people of Uzbek nationality.
Materials and methods: we performed screening for DM and prediabetes among urban and rural population of Uzbek nationality aged 35 and older, in 3 regions of the Republic of Uzbekistan. 2159 people were screened (67.8\% - women). Screening consisted of two stages: 1) filling in the questionnaire of DM based on the FINDRISK; 2) oral glucose tolerance test (OGTT) with $75 \mathrm{~g}$ of glucose to all individuals regardless of the assessed risk. DM and prediabetes were diagnosed according to IDF recommendations.
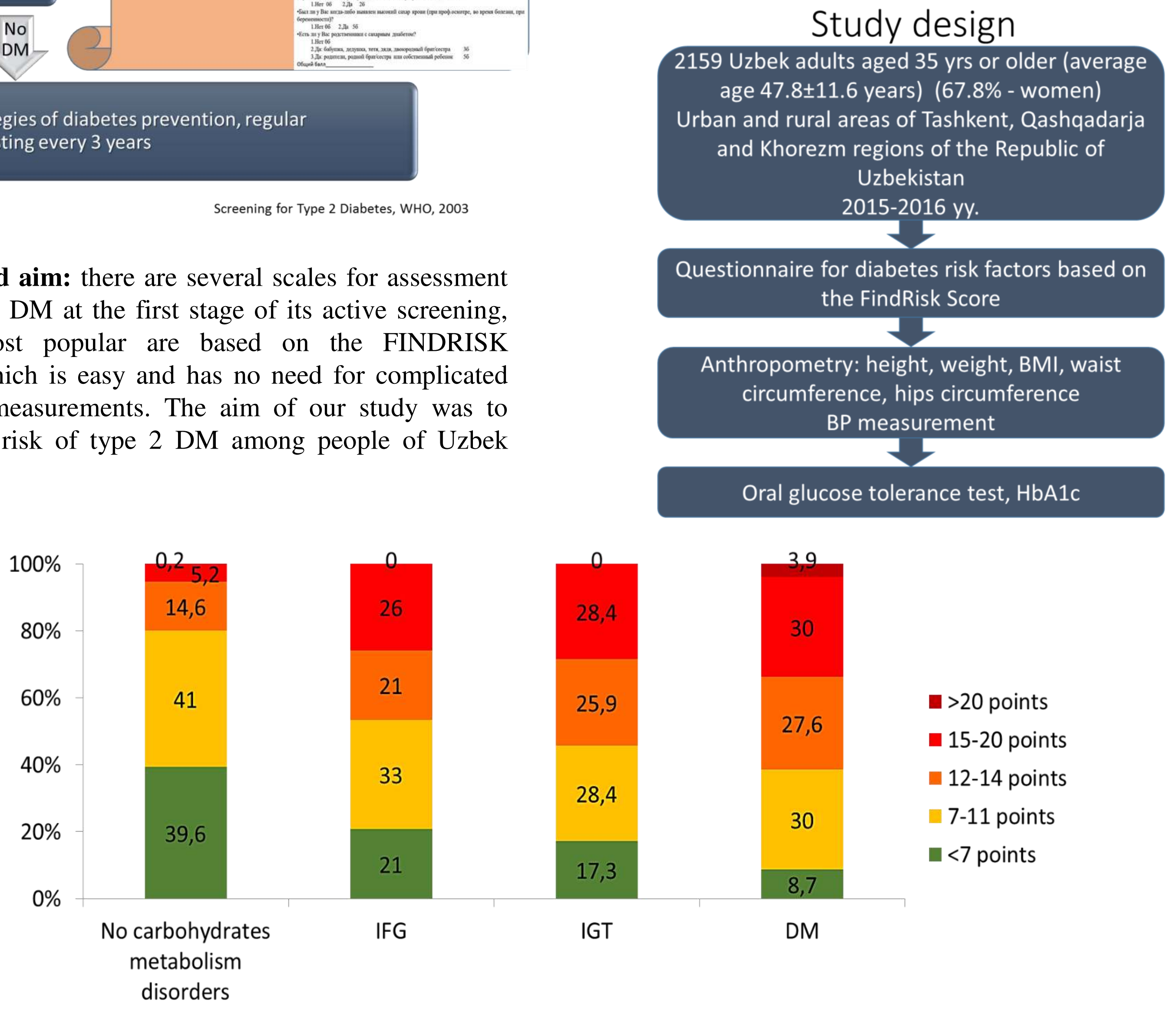

Results: among people without carbohydrate metabolism disorders, $94.6 \%$ had increased and moderate risk (score up to 14 points). Among people with IFG, only $25 \%$ had high DM risk (15 to 20 points), $21 \%$ had moderate risk, 33\% - increased risk ( 7 to 11 points), and $21 \%$ - low risk ( $<7$ points). Among people with IGT, only $24.8 \%$ had high DM risk, $25.9 \%$ had moderate risk, $28.4 \%$ - increased risk, and $17.3 \%$ - low risk. Among people with DM diagnosed during the screening, only $33.9 \%$ had high (30\%) and very high (3.9\%) DM risk, whereas $27.6 \%$ had moderate risk, $30 \%$ - increased risk, and $8.7 \%$ - low risk of type $2 \mathrm{DM}$.
Conclusion: during active screening for type 2 DM among people of Uzbek nationality, OGTT performed for only individuals with high and very high DM risk according to the FINDRISK questionnaire would have miss reveal of $66.3 \%$ of patients with diabetes. Probably, it is necessary to use anthropometric references specific for Uzbek nation, and also to perform integral assessment of type $2 \mathrm{DM}$ risk factors for people of Uzbek nationality. 